\title{
Message from the WDES 2016 Workshop Chairs
}

It is a great pleasure for the WDES organizers to this exciting workshop that will provide researchers and practitioners with a forum to exchange ideas and experiences, analyze research \& development issues, discuss promising solutions, and propose new directions in the field of achieving dependability in evolving systems.

Modern systems are growing in complexity and size, and their architectures and interactions between components are not static, but rather subject to change due to planned or unplanned events; in general, their environment is dynamic and uncertain.

Many systems on which our nowadays society depends are increasingly subject to changes during their lifetime, especially infrastructures that are meant to be in place for several years. Short-term changes, frequent and with immediate effect in terms of offered services, built-in structure, and interactions with other entities, are referred to as system dynamicity. Longterm changes, in terms of functions added or modified, to adapt to new standards and regulations, are referred to as system evolution. Adaptivity is a key feature to deal with dynamicity and evolution. In response to their perception of the environment and the system itself, systems should be capable of adjusting their structure and behavior in a predictable manner.

At the same time, many of such systems are safety-critical, or have strong performance or dependability requirements. This poses new challenges in the development of adaptation techniques, as the outcomes of adaptation must not violate dependability and safety requirements. New approaches are needed both at design time - to handle system complexity, document adaptation facilities, and keep track of relevant information - as well as at run-time - to evaluate adaptation alternatives, improve the perception of the system and environment, and document the occurring adaptation. Furthermore, adaptation should address changes occurring during systems' lifetime by both perspectives: dynamicity and evolution.

This edition of WDES focuses on the theme of adaptation to face dynamicity and evolution in dependable complex systems. The accepted papers address several aspects of dependability, including privacy, security, and maintenance, and span different kinds of systems, including mobile systems, mission-critical systems, and power infrastructures. The technical sessions will be complemented by a keynote speech closely related with the theme of the workshop.

WDES 2016 is co-located with the 2016 Latin-American Dependable Computing Conference (LADC 2016), and will take place in October 19-21, 2016 in the beautiful city of Cali, hosted by Universidad San Buenaventura de Cali. The Workshop organizers would like to thank the precious support received from LADC Organizers and Committee members, in 
particular the General Chair Julio Ariel Hurtado Alegria and the Program Committee Chair Regina Moraes. A special appreciation goes to all the WDES Program Committee members for their contribution to the realization of the Workshop.

Last but not least, the organizers would like to acknowledge the support offered to WDES by the DEVASSES (DEsign, Verification and VAlidation of large scale, dynamic Service SystEmS) FP7 Research Project, and by the Brazilian CNPq and CAPES agencies.

We sincerely hope all attendees will profit from the workshop technical activities and will exploit the opportunity of meeting each other and discovering the beauty of Cali, one of the most culturally rich sites of Colombia.

Leonardo Montecchi, University of Firenze, Italy

Ivan Mura, Los Andes University, Colombia

Baldoino Neto, Universidade Federal de Alagoas, Brazil

Cecilia Rubira, Universidade Estadual de Campinas, Brazil

WDES 2016 Workshop Chairs 